\title{
Catabolite Repression of Pseudomonas aeruginosa Amidase: The Effect of Carbon Source on Amidase Synthesis
}

\author{
By P. F. SMYTH AND PATRICIA H. CLARKE \\ Department of Biochemistry, University College London, London WCIE $6 B T$
}

(Received 2 January I975; revised 17 March I975)

SUMMAR Y

Synthesis of the Pseudomonas aeruginosa aliphatic amidase was repressed severely by succinate and malate and less severely by glucose, acetate or lactate. Amidase synthesis in inducible and constitutive strains was stimulated by cyclic AMP, which also gave partial relief to catabolite repression produced by the addition of lactate to cultures growing in pyruvate medium. Mutants which were resistant to catabolite repression were isolated from succinate + lactamide medium.

\section{INTRODUCTION}

Most Pseudomonas species have a remarkable nutritional versatility (Stanier, Palleroni \& Doudoroff, 1966) and can metabolize a wide range of compounds by convergent catabolic pathways. The synthesis of catabolic enzymes is controlled by sequential induction of groups of enzymes by substrates or intermediates of the pathway and by repression by later products (Stevenson \& Mandelstam, 1965; Ornston, 1971; Higgins \& Mandelstam, 1972). In Escherichia coli, glucose is known to produce marked catabolic repression of the synthesis of inducible enzymes such as $\beta$-galactosidase. The glucose effect is now thought to be due to a lowering of the level of cyclic AMP (c-AMP) in the cell. A positive control for catabolic genes involves the interaction of c-AMP with an activator protein, which then binds at the promoter site of a catabolite repression-sensitive operon and facilitates the initiation of transcription (De Crombrugghe et al. 1971).

Pseudomonas aeruginosa utilizes citrate in preference to glucose ( $\mathrm{Ng}$ \& Dawes, 1973). Succinate and other intermediates of the tricarboxylic acid cycle are in general more effective than glucose in repressing the synthesis of inducible enzymes of $P$. aeruginosa. This may be related to the aerobic nature of this organism and the predominance of the tricarboxylic acid cycle as the terminal sequence of most of the convergent catabolic pathways. Brammar \& Clarke (1964) showed that amidase synthesis is subject to catabolite repression, and in this and the following paper we describe the effects of various compounds on amidase synthesis in the wild-type $P$. aeruginosa strain PACI and in mutants with alterations in regulator or structural genes concerned with amidase synthesis or activity.

\section{METHODS}

Bacterial strains. The $P$. aeruginosa strains used in this investigation are listed in Tables I and 5. The series numbers denote the class of amidase mutant and its derivation, e.g. CIILI is a catabolite repression-resistant mutant derived from the constitutive strain CII. Since the strains described have a range of different amide growth phenotypes it is convenient to use this shorthand nomenclature to indicate their relationships to one another. 
Table I. Strains of Pseudomonas aeruginosa used in this study

(See also Table 5.)

\begin{tabular}{lcll} 
Strain no. & Series no. & Relevant phenotype* & \multicolumn{1}{c}{ Reference } \\
PACI & WT & AmiA Ind & - \\
PACIOI & CI & AmiA Con But-r & Brammar t al. (1967) \\
PACI I I & CI I & AmiA Con But-s & Brown \& Clarke (1970) \\
PACI42 & LIO & AmiA Con But-r Crp-r & Brown \& Clarke (I970) \\
PAC35I & B6 & AmiB Con But-s & Brown et al. (1969) \\
PAC509 & At9 & AmiA Ind Ace & Skinner \& Clarke (1968) \\
PAC566 & - & AmiB Con Ace & This paper
\end{tabular}

* Phenotype abbreviations: AmiA, wild-type amidase; AmiB, mutant B amidase; Ind, inducible; Con, constitutive; But-s and But-r, sensitive or resistant to repression by butyramide; Crp-r, resistant to catabolite repression by succinate; Ace $^{-}$, defective in acetic thiokinase.

Bacteriophage FI 6 (Holloway, Egan \& Monk, I960) was provided by Professor B. W. Holloway.

Media. Cultures were maintained on Oxoid nutrient agar. Minimal salt media containing various amides were prepared as described by Brammar, Clarke \& Skinner (1967), and Brown, Brown \& Clarke (I969).

Growth conditions. Small volumes were grown in $5 \mathrm{ml}$ of nutrient broth or minimal salt medium in Universal screw-cap bottles. Larger volumes were inoculated with $2 \%(\mathrm{w} / \mathrm{v})$ of an overnight culture in the same medium and grown in conical flasks of capacity at least five times the medium volume. Bacterial dry weight was determined by relating the extinction at $670 \mathrm{~nm}$ to a standard curve. Differential rates of amidase synthesis were measured in cultures which had reached the exponential phase of growth. Inducer was added if necessary and after a suitable period ( 30 to $60 \mathrm{~min}$ after the addition of inducer) the culture was split into the required number of pre-warmed flasks, some containing the test compound and one acting as a control. Inducer and compounds being tested as repressors were added to give a final concentration of $10 \mathrm{mM}$. Samples ( $\mathrm{I} \mathrm{ml}$ ) were removed at intervals and kept on ice until the end of the experiment. All cultures were grown at $37^{\circ} \mathrm{C}$.

Cyclic AMP experiments. All these experiments were carried out with small volumes of culture. The exponentially-growing cultures $(4$ to $6 \mathrm{ml})$ were divided between two or three $25 \mathrm{ml}$ conical flasks and incubated in a shaking water bath. Samples (0.1 $\mathrm{ml})$ were removed and assayed directly. Cyclic AMP was neutralized with $\mathrm{NaOH}$ just before it was added to the growing bacteria. Adenosine cyclic $3^{\prime}, 5^{\prime}$-monophosphoric acid was obtained from Sigma.

Amidase assays. The enzyme was assayed by the transferase reaction using the method of Brammar \& Clarke (1964) modified when necessary according to the size and activity of the sample.

\section{RESULTS}

The effect of carbon source on growth rate and amidase synthesis

Amidase can be induced by substrate amides, e.g. acetamide, or non-substrate amides, e.g. $\mathrm{N}$-acetylacetamide, in minimal salt medium containing succinate or pyruvate as the major carbon source for growth. The differential rate of synthesis by the wild-type strain PACI, induced by $\mathrm{N}$-acetylacetamide, during exponential growth in pyruvate medium is 4- to 5 -fold greater than that in succinate medium. When succinate (IO mM) is added to cultures growing in pyruvate medium, which have already been induced, the synthesis of amidase is reduced by more than $90 \%$ within $3 \mathrm{~min}$. This rapid repression of amidase 
Table 2. The effect of carbon compounds on amidase synthesis by Pseudomonas aeruginosa

Cultures were grown in I \% pyruvate-minimal salt medium. For strain PACI, $N$-acetylacetamide was added when the culture had reached a cell density of $0.1 \mathrm{mg}$ bacteria $/ \mathrm{ml}$ and the repressing compound was added after about $30 \mathrm{~min}$. All additions gave a final concentration of $10 \mathrm{~mm}$. Enzyme activity was measured in samples taken over at least $2 \mathrm{~h}$.

\begin{tabular}{|c|c|c|c|c|c|}
\hline \multirow[b]{3}{*}{$\begin{array}{c}\text { Added } \\
\text { metabolite }\end{array}$} & \multicolumn{2}{|c|}{ Mean generation time } & \multirow{2}{*}{\multicolumn{3}{|c|}{ Repression of amidase synthesis ( $\%)$}} \\
\hline & & & & & \\
\hline & $\begin{array}{c}\text { After } \\
\text { addition }\end{array}$ & $\begin{array}{l}\text { metabolite } \\
\text { alone }\end{array}$ & $\begin{array}{c}\text { PACI } \\
\text { (inducible) }\end{array}$ & $\begin{array}{c}\text { PACIOI } \\
\text { (constitutive) }\end{array}$ & $\begin{array}{c}\text { PACI I I } \\
\text { (constitutive) }\end{array}$ \\
\hline - & $\mathrm{I} \cdot 25$ & $I \cdot 25$ & - & - & - \\
\hline Malate & 0.7 & ND & $95-100$ & $95-100$ & $95-100$ \\
\hline Succinate & 0.75 & 0.75 & $85-95$ & $85-95$ & $85-95$ \\
\hline Acetate & 0.9 & $I \cdot 0$ & 65 & 95 & 85 \\
\hline Glycerol & 0.9 & $3 \cdot 5$ & 50 & ND & ND \\
\hline Lactate & 0.9 & $I \cdot 0$ & $45-55$ & ND & $45-55$ \\
\hline Glucose & 0.9 & $1 \cdot 2$ & $30-35$ & $30-35$ & $30-50$ \\
\hline Citrate & 0.85 & $I \cdot 2$ & 30 & ND & ND \\
\hline
\end{tabular}

synthesis occurs also with mutants which synthesize amidase constitutively, so cannot be due to inhibition of inducer uptake. The rapid and severe repression produced by the addition of succinate to cultures growing in pyruvate medium is similar to the severe transient repression of $\beta$-galactosidase synthesis produced by glucose in $E$. coli induced with isopropyl- $\beta$-D-thiogalactoside in glycerol medium (Moses \& Prevost, I966).

Many other compounds were found to repress amidase synthesis in pyruvate medium, and this was usually accompanied by an increase in the growth rate of the culture (Table 2). Glycerol, which supports a mean generation time of $3.5 \mathrm{~h}$ when present as the sole carbon source, was able to reduce the mean generation time of the culture from $\mathrm{I} \cdot 25$ to $0.9 \mathrm{~h}$ with a $50 \%$ reduction in the differential rate of amidase synthesis. Succinate and malate were the most effective repressing compounds for the inducible wild-type strain and for the constitutive mutants PACIOI and PACI I I. Although succinate has the effect of reducing the rate of amidase synthesis of pyruvate-grown cultures to a very low level, it must be emphasized that when cultures have been grown throughout in succinate medium the differential rate of amidase synthesis is reasonably high, reaching about $20 \%$ of that in pyruvate medium. Brammar \& Clarke (I964) found that repression of amidase induction in succinate medium by propionate could be partially relieved by the addition of higher concentrations of acetamide as inducer. It was clear that the repressing compounds were not exerting a specific effect on amidase synthesis but were affecting it indirectly by disturbing the metabolic balance of the cell.

Transient catabolite repression of $\beta$-galactosidase by glucose continues for 0.1 to 0.5 of a generation and is followed by a long period of less severe repression (Moses \& Prevost, 1966). Repression of amidase synthesis in pyruvate medium by Io mM-succinate continues for several hours, but if succinate is added at lower concentrations then the rate of synthesis returns to a high rate as soon as the succinate has been utilized (Fig. I). Repression is as severe with $2.5 \mathrm{~mm}$ - and $5 \mathrm{~mm}$-succinate as with $10 \mathrm{mM}-$, but the duration of repression is much shorter. At the end of the period of severe repression the culture returns to a rate of amidase synthesis higher than the control rate. 

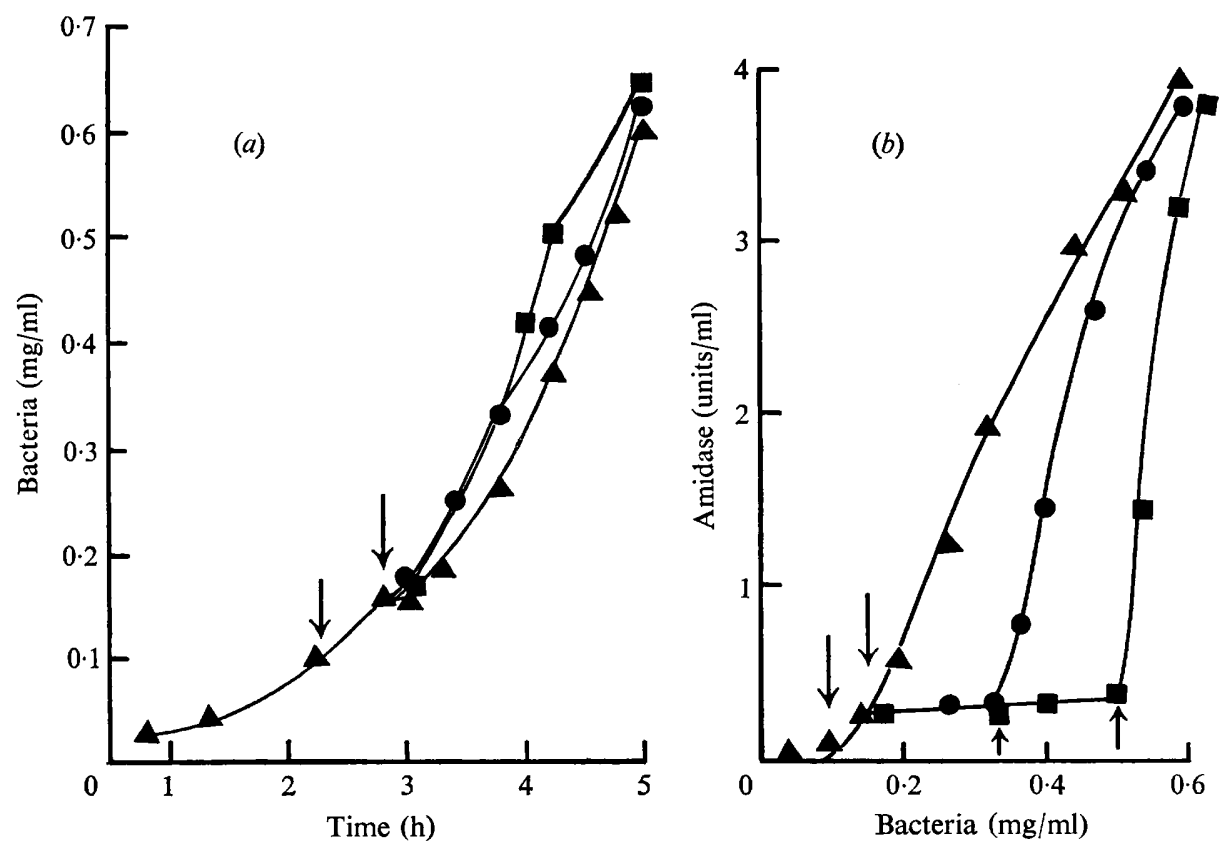

Fig. I. Effect of succinate on $(a)$ growth and $(b)$ amidase synthesis of strain PACI in pyruvate medium. $N$-acetylacetamide (IO mM) was added at the time indicated by first arrow. At the time indicated by the second arrow the culture was divided between three flasks: $\boldsymbol{\Delta}$, control; $\boldsymbol{\theta},+2.5 \mathrm{mM}-$ succinate; $\boldsymbol{\square},+5 \mathrm{~mm}$-succinate. The lower arrows in $(b)$ indicate when change in growth rate occurred.
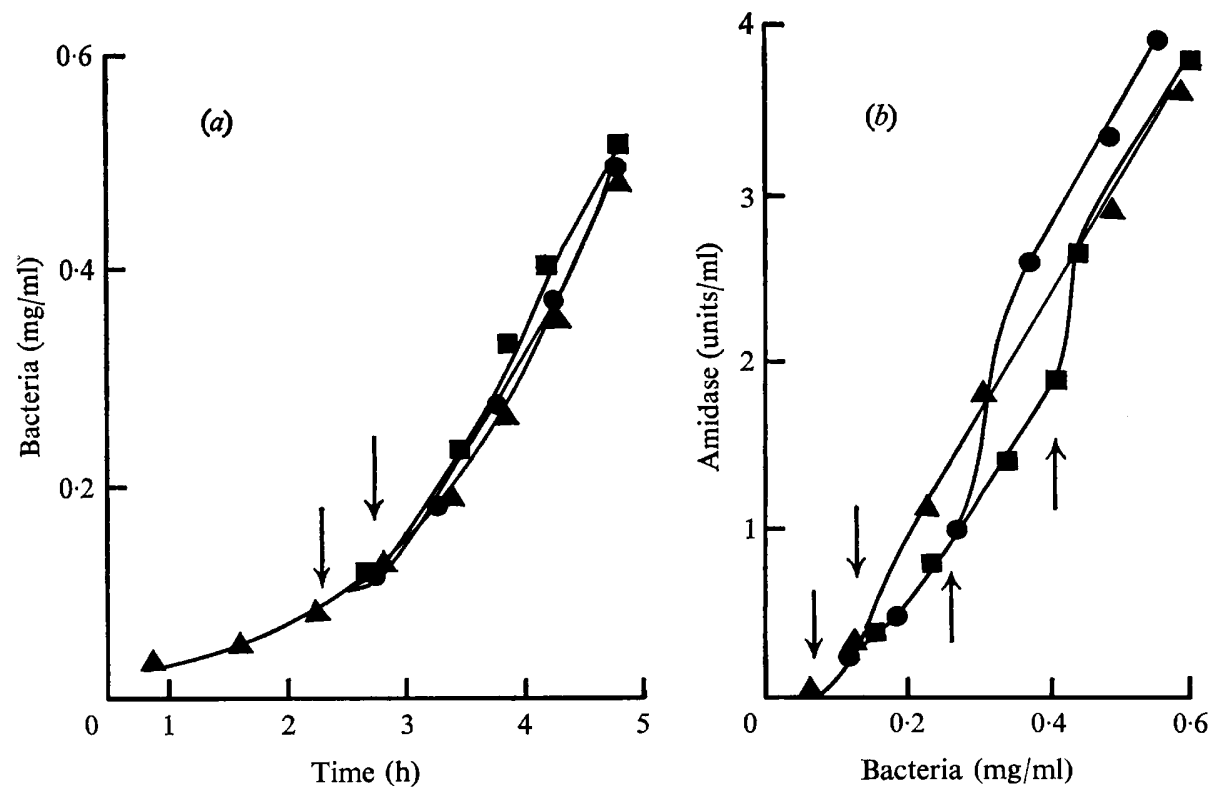

Fig. 2. Effect of glucose on $(a)$ growth and $(b)$ amidase synthesis of strain PACI in pyruvate medium. Acetamide (IO $\mathrm{mM}$ ) was added at the time indicated by first arrow. At the time indicated by the second arrow the culture was divided between three flasks: $\boldsymbol{\Delta}$, control; $\boldsymbol{0},+0.5 \mathrm{~mm}$-glucose; $\square,+\mathbf{I} \cdot 0$ mM-glucose. The lower arrows in $(b)$ indicate when changes in growth rate occurred. 
Table 3. Induction and repression of amidase synthesis by acetamide

Cultures were grown in I \% pyruvate-minimal salt medium.

\begin{tabular}{lcccc} 
Strain no. & Series no. & $\begin{array}{c}\text { Addition of } \\
\text { 20 }\end{array}$ & $\begin{array}{c}\text { Differential } \\
\text { rate }\end{array}$ & $\begin{array}{c}\text { Mean generation time } \\
(\mathrm{h})\end{array}$ \\
PACI & WT & - & 0 & $\mathrm{I} \cdot 25$ \\
\multirow{2}{*}{ PACIII } & & + & 6 & 0.95 \\
& CII & - & 45 & $\mathrm{I} \cdot \mathrm{I}$ \\
PAC509 & & + & 6 & 0.8 \\
& At9 & - & 0 & $\mathrm{I} \cdot 2$ \\
& & + & 47 & $\mathrm{I} \cdot 2$
\end{tabular}

Similar results were obtained for amidase synthesis with glucose as the repressing compound. Glucose gave only $30 \%$ repression of amidase synthesis, and this was the same whether the concentration of glucose was $0.5, \mathrm{I} \cdot 0$ or $10 \mathrm{~mm}$. With the lower concentrations the period of $30 \%$ repression was followed by a brief overshoot period, when the rate of synthesis was higher than the control, and finally the culture returned to the same differential rate as the control (Fig. 2).

\section{The need to metabolize the repressing compound to produce an effect}

The experiments with succinate and glucose indicated that the repressing compound only produced its effect on amidase synthesis while it was being metabolized. Since acetate was a fairly good catabolite repressor when it was added to the pyruvate-grown cultures, it was thought that an inducible strain, which could metabolize acetamide and acetate, would be induced by acetamide and repressed by the acetate formed as the result of enzyme action. Constitutive strains would also be expected to be repressed by acetamide in the same way and this effect was expected to be most marked in pyruvate medium. Table 3 shows that the constitutive strain PACII I was repressed by acetamide and that the repressed rate was the same as that of the wild type induced by acetamide. Strain PAC509 is an amidase-inducible strain which is defective in acetic thiokinase and cannot grow on acetamide or acetate as carbon source. This strain was induced by acetamide to the same rate of amidase synthesis as that of the constitutive strain PACIII in the absence of acetamide. This experiment indicated very clearly that acetate had to be metabolized to produce the repressing effect, but was complicated by the necessity to induce amidase synthesis in strain PAC509. A new strain, PAC566, was constructed which was constitutive for the synthesis of a mutant amidase. This carried the acetic thiokinase mutation, a mutation in the amiR gene conferring constitutivity and a mutation in the amiE gene determining the mutant B amidase (Brown et al. 1969). It was constructed by transducing the B amidase mutations from PAC35 I (B6) into strain PAC509. The transductants could be selected on butyramide plates, since although they were unable to grow on acetamide or acetate they could grow on butyramide or butyrate. Table 4 shows that while strain PAC35I, which can grown on acetamide and acetate, is as sensitive as the constitutive strain PACII I to repression by acetate, the acetic thiokinase mutant PAC566 is not repressed by either acetate or acetamide. We conclude that acetate must be metabolized to produce catabolite repression.

\section{Isolation of catabolite repression-resistant mutants}

Lactamide is a good inducer of amidase synthesis but is a very poor substrate. It is possible to select mutants which have varying degrees of resistance to catabolite repression 
Table 4. Catabolite repression by succinate, acetate and acetamide

Cultures were grown in $1 \%$ pyruvate-minimal salt medium. $N$-acetylacetamide (10 mM) was added to the culture of PAC509, as inducer; no inducer was added to other strains.

$\begin{array}{ccccc}\text { Strain no. } & \text { Series no. } & \overbrace{\begin{array}{c}\text { Succinate } \\ \text { (IO mM) }\end{array}}^{\text {Repression (\%) }} & \begin{array}{c}\text { Acetate } \\ \text { (IO mM) }\end{array} & \begin{array}{c}\text { Acetamide } \\ \text { (IO mM) }\end{array} \\ \text { PACI I } & \text { CI I } & 85 & 85 & 85 \\ \text { PAC35 I } & \text { B6 } & 83 & 82 & 82 \\ \text { PAC509 } & \text { At9 } & 94 & 0 & - \\ \text { PAC566 } & \text { B6/At9 } & 82 & 0 & 0\end{array}$

Table 5. Comparison of amidase specific activities of mutant strains of P. aeruginosa grown overnight in succinate medium with repression by succinate in pyruvate medium

\begin{tabular}{|c|c|c|c|c|}
\hline Strain no. & Series no.* & Parent & $\begin{array}{l}\text { Repression of } \\
\text { differential rate by } \\
\text { succinate }(\%)\end{array}$ & $\begin{array}{l}\text { Specific activity after } \\
\text { overnight growth in } \\
\text { succinate medium }\end{array}$ \\
\hline PACIOI, PACI I I & CI, CI I & PACI & $85-95$ & I 2-24 \\
\hline PACI 42 & LIO & PACI & & \\
\hline PACI 44, PACI 45 & CILI, CIL2 & PACIOI & $70-75$ & $36-70$ \\
\hline PACI46, PACI 47 & $\mathrm{CIL}_{3} \mathrm{CIL} 7$ & PACIOIJ & & \\
\hline PACI 48 & CIL8 & PACIOI & $50-60$ & $72-100$ \\
\hline$\dagger$ & CIILI-CIIL39 & PACI I I & ND & $48-70$ \\
\hline
\end{tabular}

ND, Not determined.

* Series CIL and CIIL isolated on S/L plates from constitutive strains CI and CI I respectively. All were constitutive but varied in sensitivity to catabolite repression.

$\dagger$ No numbers assigned.

of amidase synthesis, from plates containing lactamide as the nitrogen source and a repressing compound such as succinate, malate or glucose as the carbon source ( $\mathrm{S} / \mathrm{L}, \mathrm{M} / \mathrm{L}$ or $\mathrm{G} / \mathrm{L}$ plates). On these media the wild-type strain PACI and most constitutive mutants grow very slowly, and mutant catabolite repression-resistant clones are readily isolated. Some of them have altered growth characteristics which are immediately obvious. For example, strain PACI5I (MI) was completely resistant to catabolite repression by succinate or malate when growing exponentially in pyruvate medium and induced by $\mathrm{N}$-acetylacetamide. The addition of succinate or malate did not result in an increase in the growth rate, as occurred with the wild-type strain (Fig. 2), and hence mutant MI probably has a mutation affecting the rates of succinate and malate metabolism.

All the mutants isolated from S/L plates showed some degree of resistance to catabolite repression when the differential rate of amidase synthesis was measured in pyruvate medium in the presence and absence of succinate. This could be correlated with the amidase specific activities of the strains after overnight growth in succinate medium. Although wide variations in the specific activities of cultures which have reached, or are about to reach, the stationary growth phase are inevitable for an enzyme whose rate of synthesis is so dependent on the metabolic state of the cell, it was found in practice that by standardizing the medium and growth conditions it was possible to get reasonably consistent values for cultures tested on many different occasions. Table 5 shows the range of values obtained for some of these mutants grown in succinate-minimal salt medium. These represent from 0.5 to $5.0 \%$ of the bacterial dry weight as amidase. Cultures grown overnight on pyruvate reach higher values 

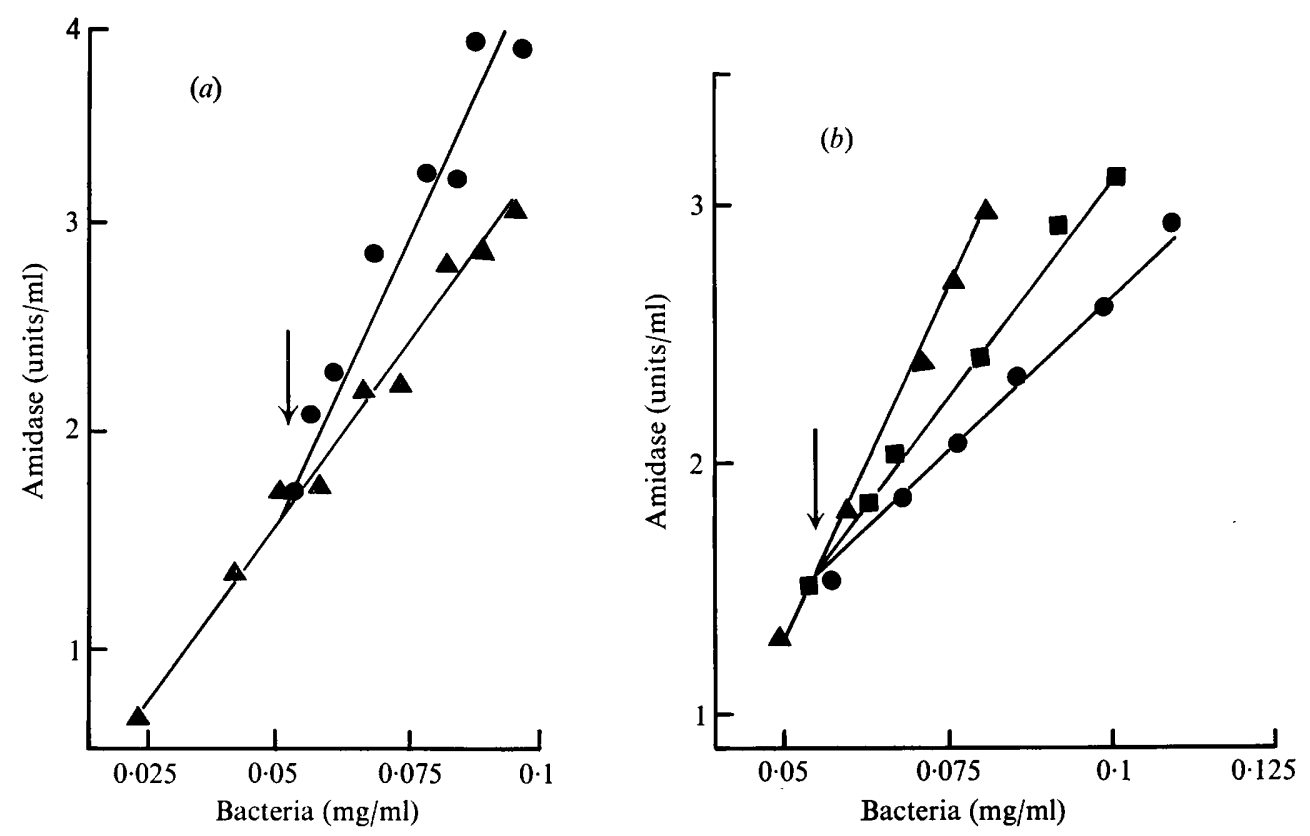

Fig. 3. Effect of cyclic $3^{\prime}, 5^{\prime}$-adenosine monophosphate on amidase synthesis and lactate repression in the constitutive strain PACI I I growing in pyruvate medium. (a) At the time indicated by the arrow the culture was divided between two flasks: $\Delta$, control; $\boldsymbol{\theta},+30$ mM-c-AMP. $(b)$ At the time indicated by the arrow the culture was divided between three flasks: $\Delta$, control; $\boldsymbol{\ominus},+$ Io mM-lactate;

$\mathbf{\square}$, + Io mM-lactate and $30 \mathrm{~mm}-\mathrm{c}-\mathrm{AMP}$.

and the specific activity of strain PACI42 (LIO) is usually greater than IOO. Although lactate represses amidase synthesis of cultures growing in pyruvate, when it is used as the sole carbon source for growth, repression is much less; lactate-grown cultures after overnight growth are similar in specific activities to those grown in pyruvate medium.

Some of the mutants isolated from the wild type on S/L plates were both resistant to catabolite repression and constitutive. Strain PACI42 was tested for linkage of these two markers but while the constitutivity marker was cotransduced with the amidase structural gene at the usual high frequency of $>90 \%$ (Brammar et al. 1967), there was no cotransduction of the catabolite repression-resistance marker. It appeared that this strain had simultaneously acquired two different mutations. Strains PACI48 (CIL8) and mutants CIILI to CIIL39 were analysed similarly and no linkage could be found between the catabolite repression-resistance marker and the constitutivity mutation for the amidase genes.

\section{The effect of $c$-AMP}

Cyclic AMP has been shown to stimulate the rate of synthesis of $\beta$-galactosidase and to overcome catabolite repression by glucose in E. coli (Pastan \& Perlman, 1968). It was thought that it might have a similar effect on inducible enzymes of $P$. aeruginosa. It was found in preliminary experiments that the severe catabolite repression of amidase synthesis produced by succinate was not relieved by $30 \mathrm{mM}$-c-AMP. There were no complicating effects of enzyme inhibition since c-AMP did not inhibit amidase activity of cell suspensions even at a concentration of $0.3 \mathrm{M}$, but it could not be ruled out that succinate may have inhibited uptake of c-AMP. Botsford \& deMoss (I97I) found that in glycerol medium 


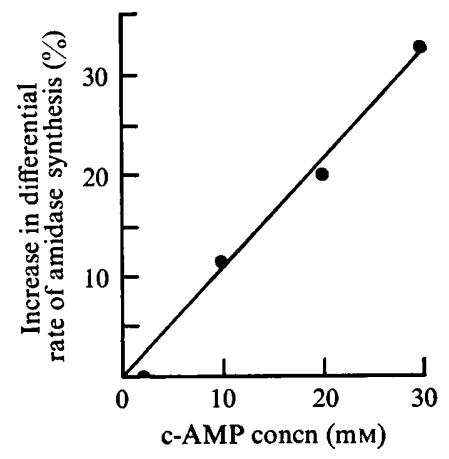

Fig. 4. Stimulation by cyclic $3^{\prime}, 5^{\prime}$-adenosine monophosphate of amidase synthesis by the constitutive strain PACI I of amidase synthesis in pyruvate medium.

c-AMP could relieve the rather weak catabolite repression of tryptophanase resulting from the addition of glucose, but not the severe repression produced by pyruvate. It was therefore thought possible that c-AMP might relieve the mild catabolite repression of amidase produced by compounds other than succinate.

Lactate produces 45 to $55 \%$ repression of amidase synthesis of the wild-type PACI and the constitutive strain PACII I grown in pyruvate medium (Table 2). Thirty mM-c-AMP stimulated the differential rate of $\mathrm{N}$-acetylacetamide-induced amidase synthesis of strain PACI and also the constitutive synthesis of strain PACI I I. This concentration of c-AMP gave partial relief of lactate repression in both strains. Figure 3 shows the behaviour of the constitutive strain PACI I I. In this particular experiment the differential rate increased by about $25 \%$ with the addition of 30 mM-c-AMP but in other experiments the increase varied from I 5 to $40 \%$. The stimulation by c-AMP was concentration-dependent and even at $30 \mathrm{~mm}$ the system was clearly not saturated (Fig. 4). This suggested that the cells were relatively impermeable to this compound, but attempts to increase cell permeability by adding EDTA were unsuccessful since this strain lyses very easily. Amidase synthesis of the inducible

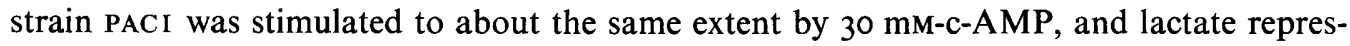
sion was relieved by about the same amount as for strain PACIII. The same experiments were carried out with another inducible strain, PAOI (Holloway, I955), and constitutive mutants derived from it, with similar results. Thus, the c-AMP effect was not strain dependent.

The level of c-AMP in the cell depends on a number of factors, one of which is the rate of breakdown by phosphodiesterases (Sutherland \& Rall, 1958). Aboud \& Burger (I97I) showed that the c-AMP diesterase of $E$. coli is inhibited by theophylline and that I mMtheophylline potentiated the stimulation of $\beta$-galactosidase synthesis by c-AMP in glycerol medium. Theophylline (I $\mathrm{mM}$ ) had a similar effect in increasing the stimulation of the rate of amidase synthesis of strain PACI I I by c-AMP. Dibutyryl c-AMP and 5'-AMP had no effect on amidase synthesis by this strain.

\section{DISCUSSION}

The term 'catabolite repression' has been used here in a very general sense to describe the effect of various carbon compounds in reducing the amount of amidase synthesized by $P$. aeruginosa. The methods used to demonstrate catabolite repression include: (i) comparison of amidase specific activities of cultures after overnight growth in succinate or 
pyruvate medium; (ii) comparison of the differential rates of amidase synthesis of cultures growing exponentially in succinate or pyruvate medium; (iii) measurements of the effects of adding succinate, or other compounds, to the rates of amidase synthesis in pyruvate medium; and (iv) observations of the growth of cultures on selective media such as $S / L$ or $M / L$ plates. Clarke, Houldsworth \& Lilly (1968) reported that cultures grown in a chemostat with acetamide as the carbon-limiting compound for growth, had a greatly reduced amidase specific activity at the higher growth rates. We consider that all these observations reflect the same underlying mechanism for the control of amidase synthesis which can be described as 'catabolite repression'. Mutants which were isolated from the selective S/L medium differed from the wild type in all the above characteristics.

It was not possible from these experiments to define at what stage of amidase synthesis the catabolite repression exerted by succinate and other compounds was having its effect. The possibility that succinate repression was entirely due to inhibition of inducer uptake was ruled out by its effect being equally marked in inducible and constitutive strains. The experiments with the acetic thiokinase-defective mutants PAC509 and PAC566 showed that acetate must be metabolized to produce repression of amidase synthesis and therefore exerts its effect indirectly. This is in contrast to the specific repression produced by acetamide analogues such as butyramide, which are thought to bind to the amidase regulator protein determined by the amiR gene without producing the correct conformation required for promoting amidase synthesis (Brown \& Clarke, 1970).

The nature of the mutations conferring resistance to catabolite repression in the mutants isolated from $\mathrm{S} / \mathrm{L}$ plates was not determined, although it was certain that for those that were tested they were not linked to the amidase structural gene. Some of the mutants had reduced rates of succinate metabolism, and these may be compared with the mutants resistant to glucose repression of $\beta$-galactosidase synthesis which were isolated by Loomis \& Magasanik (1965) from plates containing glucose as the carbon source and $N$-acetyl-lactosamine as the nitrogen source. It was later found that these were not specific for the lac operon and were probably defective in glucose metabolism. We have not yet identified among the $\mathrm{L}$ series of mutants any which are derepressed for other inducible $P$. aeruginosa enzymes. Brill \& Magasanik ( 1969) have isolated mutants of Salmonella typhimurium which are derepressed for several catabolic enzymes and these too were defective in glucose metabolism.

The kinetics of onset of succinate repression in exponentially-growing cultures was consistent with an effect at the transcriptional level, with maximum repression being reached about 3 min after the addition of succinate (Brammar, 1965). The synthesis of amidase in both inducible and constitutive strains was stimulated by c-AMP and repression resulting from the addition of lactate was partially relieved. It seems likely that amidase is subject to the same general control as has been described for many of the catabolic genes of $E$. coli. In the following paper we describe the isolation of amidase promotor mutants which are resistant to catabolite repression and show little or no response to c-AMP.

\section{REFERENCES}

Aboud, M. \& Burger, M. (197I). Cyclic 3',5' adenosine monophosphate-phosphodiesterase and the release of catabolite repression of $\beta$-galactosidase by exogenous cyclic $3^{\prime}, 5^{\prime}$ adenosine monophosphate in Escherichia coli. Biochemical and Biophysical Research Communications 43, 174-182.

Botsford, J. L. \& DeMoss, R. D. (1971). Catabolite repression of tryptophanase in Escherichia coli. Journal of Bacteriology 105, 303-3I 2.

Brammar, W. J. (1965). The control of amidase synthesis in Pseudomonas aeruginosa by induction and repression mechanisms. Ph.D. thesis, University of London. 
Brammar, W. J. \& Clarke, P. H. (I964). Induction and repression of Pseudomonas aeruginosa amidase. Journal of General Microbiology 37, 307-319.

Brammar, W. J., Clarke, P. H. \& Skinner, A. J. (1967). Biochemical and genetic studies with regulator mutants of the Pseudomonas aeruginosa amidase system. Journal of General Microbiology 47, 87-I02.

BRILL, W. \& MAGASANIK, B. (1969). Genetic and metabolic control of histidase and urocanase in Salmonella typhimurium, strain 15-59. Journal of Biological Chemistry 244, 5392-5402.

Brown, J. E., Brown, P. R. \& Clarke, P. H. (1969). Butyramide-utilizing mutants of Pseudomonas aeruginosa which produce an amidase with altered substrate specificity. Journal of General Microbiology 57, $273-285$.

Brown, J. E. \& Clarke, P. H. (I970). Mutations in a regulator gene allowing Pseudomonas aeruginosa 8602 to grow on butyramide. Journal of General Microbiology 64, 329-342.

Clarke, P. H., Houldsworth, M. A. \& Lilly, M. D. (I968). Catabolite repression and the induction of amidase synthesis by Pseudomonas aeruginosa 8602 in continuous culture. Journal of General Microbiology 51, 225-234.

De Crombrugghe, B., Chen, B., Anderson, W., Nissley, P., Gottesman, M., Pastan, I. \& Perlman, R. L. (197I). Lac DNA, RNA polymerase and cyclic AMP receptor protein, cyclic AMP, lac repressor and inducer are the essential elements for controlled lac transcription. Nature New Biology 230, I39-I42.

Higgins, S. J. \& Mandelstam, J. (I972). Regulation of pathways degrading aromatic substrates in Pseudomonas putida. Enzymic response to binary mixtures of substrates. Biochemical Journal 126, 901-916.

Holloway, B. W. (1955). Genetic recombination in Pseudomonas aeruginosa. Journal of General Microbiology 13, 572-581.

Holloway, B. W., Egan, J. B. \& Monk, M. (1960). Lysogeny in Pseudomonas aeruginosa. Australian Journal of Experimental Biology 38, 321-330.

Loomis, W. F. \& Magasanik, B. (I965). Genetic control of catabolite repression of the lac operon in Escherichia coli. Biochemical and Biophysical Research Communications 20, 230-234.

Moses, V. \& Prevost, C. (1966). Catabolite repression of $\beta$-galactosidase synthesis in Escherichia coli. Biochemical Journal roo, 336-353.

NG, F. M.-W. \& DAwEs, E. A. (I973). Chemostat studies on the regulation of glucose metabolism in Pseudomonas aeruginosa by citrate. Biochemical Journal 132, 129-140.

ORnSton, L. N. (197I). Regulation of catabolite pathways in Pseudomonas. Bacteriological Reviews 35 , 87-II6.

Pastan, I. \& Perlman, R. L. (1968). The role of the lac promotor locus in the regulation of $\beta$-galactosidase synthesis by cyclic $3^{\prime}, 5^{\prime}$-adenosine monophosphate. Proceedings of the National Academy of Sciences of the United States of America 6r, 1336-1342.

Skinner, A. J. \& Clarke, P. H. (I968). Acetate and acetamide mutants of Pseudomonas aeruginosa 8602. Journal of General Microbiology 50, 183-194.

Stanier, R. Y., Palleroni, N. J. \& Doudoroff, M. (I966). The aerobic pseudomonads: a taxonomic study. Journal of General Microbiology 43, I59-27I.

Stevenson, I. L. \& Mandelstam, J. (I965). Induction and multisensitive end-product repression in two converging pathways degrading aromatic substances in Pseudomonas fuorescens. Biochemical Journal 96, 354-362.

Sutherland, E. W. \& Rall, T. W. (1958). Fractionation and characterization of a cyclic adenine ribonucleotide formed by tissue particles. Journal of Biological Chemistry 232, I077-109I. 